\title{
Nodo integrador de la información geográfica del Agro: Geoportal IDE MINAGRI de Chile
}

\author{
María Graciela Barrera Vielma* \\ Iván Libaque Terrones*
}

Recibido 14 de marzo de 2017; aceptado 22 de mayo de 2017

\begin{abstract}
The Spatial Data Infrastructure of the Chilean Ministry of Agriculture (IDE MINAGRI), brings together the geographical information and the geoservices of the Ministry in a single interoperable on-line technological platform. Through this platform, the consolidated and standardized geographical information is made available to users. This information is the spatial expression of the Ministry's intervention at policy, plan and program levels. The objective is to facilitate decision making, mainly between ministerial authorities and agricultural professionals. The IDE MINAGRI is visible through its geoportal (<http://ide.minagri.gob.cl $>$ ) and the responsibility for coordination, continuous improvement and technical execution rests with the Natural Resources Information Center (CIREN).
\end{abstract}

Key words: Geoportal, SDI, thematic SDI, Ministry of Agriculture, Chile.

\section{Resumo}

A Infraestrutura de Dados Espaciais do Ministério da Agricultura (IDE MINAGRI) do Chile, reúne a informação geográfica e serviços do Ministério em uma única plataforma tecnológica on-line interoperável. Através desta plataforma se põe a disposição dos usuários informação geográfica consolidada e padronizada, sendo a expressão espacial da intervenção do ministério a nível de políticas planas e programas, para fazer gestão e facilitar a tomada de decisões, principalmente entre autoridades ministeriais e profissionais do agro. A IDE MINAGRI se faz visível através de seu geoportal (<http://ide.minagri.gob.cl $>$ ) e, o responsável pela coordena-

* $\quad$ Centro de Recursos Naturales (CIREN), Av. Manuel Montt 1164, Providencia Santiago, Chile, correos electrónicos: mbarrera@ciren.cl, ilibaque@ciren.cl 
ção, melhora contínua e execução técnica é do Centro de Informação de Recursos Naturais (CIREN).

Palavras chave: Geoportal, IDE, IDE temática, Ministério da Agricultura, Chile.

\section{Resumen}

La Infraestructura de Datos Espaciales del Ministerio de Agricultura (IDE MINAGRI) de Chile, reúne a la información geográfica y servicios del Ministerio en una única plataforma tecnológica on-line interoperable. A través de esta plataforma se pone a disposición de los usuarios información geográfica consolidada y estandarizada, siendo la expresión espacial de la intervención del ministerio a nivel de políticas planes y programas, para gestionar y facilitar la toma de decisiones, principalmente entre autoridades ministeriales y profesionales del agro. La IDE MINAGRI se hace visible a través de su geoportal ( $<$ http://ide.minagri.gob.cl $>$ ) y el responsable de la coordinación, mejora continua y ejecución técnica es el Centro de Información de Recursos Naturales (CIREN).

Palabras clave: Geoportal, IDE, IDE temática, Ministerio de Agricultura, Chile.

\section{IDE MINAGRI, geoportal orientado al Agro}

La Infraestructura de Datos Espaciales del Ministerio de Agricultura (IDE MINAGRI), surge como proyecto en el año 2010 ante la necesidad de contar con un sistema unificado que permitiera, a los profesionales del Ministerio, tener acceso a una base común y estandarizada de información geográfica (IG). Un sistema para gestionar la IG del ministerio y ofrecer un soporte para la toma de decisiones, principalmente a nivel de autoridades ministeriales y profesionales vinculados al ámbito agrícola y ganadero.

La IDE MINAGRI es un sistema de gestión de datos e IG interoperable, por lo tanto, sus procesos de producción se realizan en base a normas, estándares o especificaciones técnicas. Este sistema proporciona los contenidos, servicios y accesos necesarios para que los usuarios puedan hacer uso de los datos e IG relacionada al mundo rural, los recursos naturales, silvo-agropecuaria, etc., proveniente de las distintas instituciones generadoras de las IG pertenecientes al Ministerio de Agricultura. El compromiso de estas instituciones y del propio ministerio han hecho y hacen posible que la IDE MINAGRI se encuentre en constante crecimiento.

Las instituciones vinculadas al ámbito agrícola y ganadero que aportan a la IDE MINAGRI son las siguientes:

- Centro de Información de Recursos Naturales (CIREN) <http://www.ciren.cl/>

- Corporación Nacional Forestal (CONAF), <http://www.conaf.cl/>.

- Fundación para la Innovación Agraria (FIA), <http://www.fia.gob.cl/>. 
- Instituto de Investigaciones Agropecuarias (INIA), <http://www.inia.cl/>

- Instituto Nacional Forestal (INFOR), <http://www.infor.gob.cl/>.

- Instituto de Desarrollo Agropecuario (INDAP), <www.indap.gob.cl>

- Comisión Nacional de Riego (CNR), <http://www.cnr.gob.cl/>

- Oficina de Estudios y Políticas Agrarias (ODEPA), <http://www.odepa.gob.cl/>

- Servicio Agrícola y Ganadero (SAG), <http://www.sag.gob.cl/>

- Comité de Seguros del Agro (AGROSEGUROS) <https://www.agroseguros. gob.cl/>.

La entrada al sistema de gestión de datos e IG interoperable IDE MINAGRI, se realiza a través del geoportal temático: <http://ide.minagri.gob.cl > (Figura 1) que da acceso a los distintos usuarios del agro (ministerial) y de la comunidad en general (público), y ofrece las siguientes funciones/operaciones básicas:

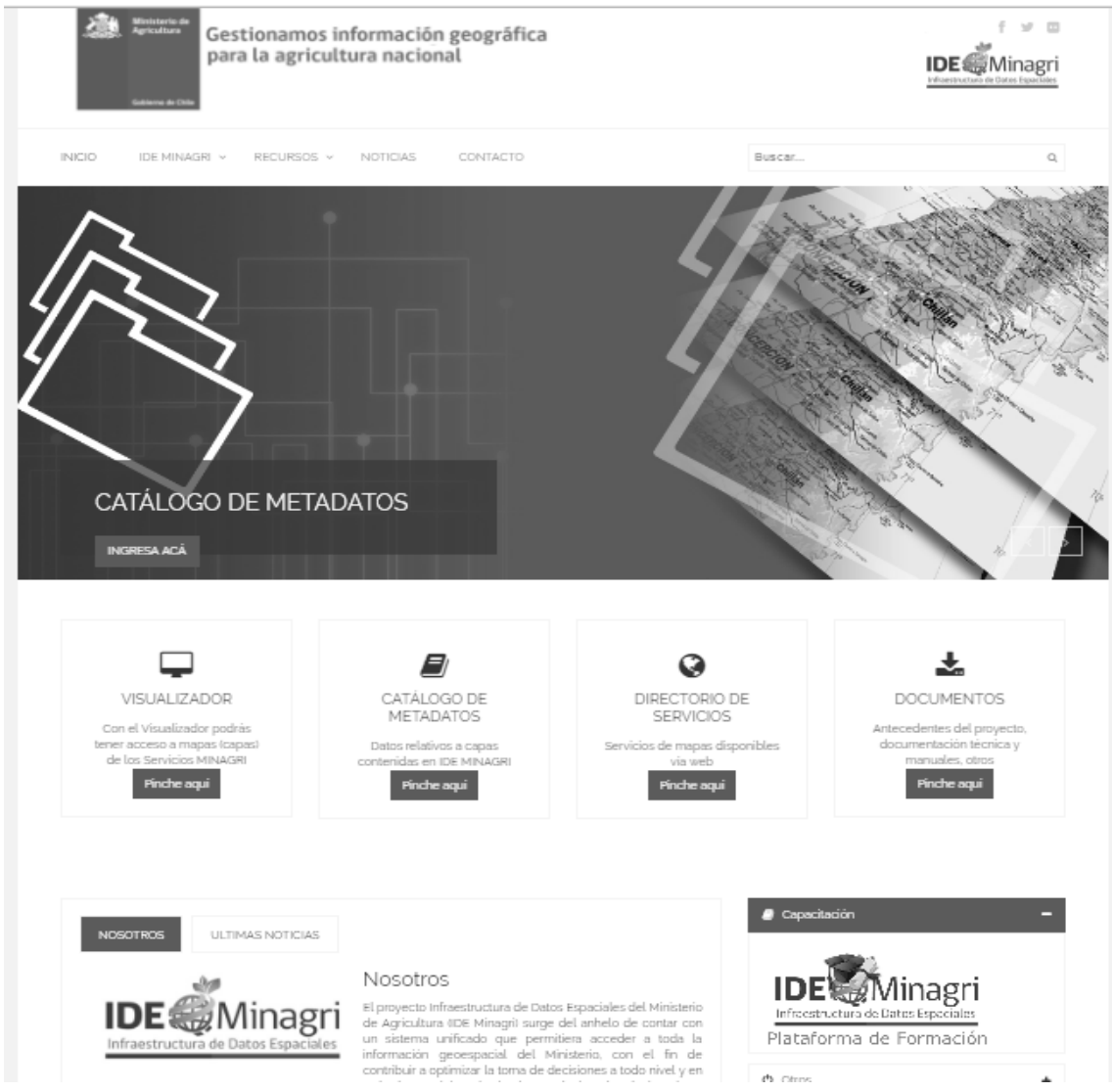

Figura 1. Vista del Geoportal IDE MINAGRI, <http://ide.minagri.gob.cl/geoweb/>. 
- Buscar/localizar: permite la búsqueda/localización través de un catálogo de metadatos implementado en Geonetwork

$<$ http://ide.minagri.gob.cl/geonetwork/srv/es/main.home>

- Visualizar: permite realizar distintos procesos, mostrar, acercarse, alejarse, moverse, superponer capas de IG, añadir servicios WMS (Web Map Service), etc. a través del visualizador de mapas <http://ide2.minagri.gob.cl/publico2/>.

Además, la IDE MINAGRI permite el acceso a datos por medio de conexiones directas a las bases de datos espaciales (BBDD) que almacenan las capas de IG de los diversos Servicios del Agro, orientado a profesionales del Ministerio con conocimientos en el manejo, análisis y procesamiento de IG.

\section{Visualizador de Mapas}

El visualizador de mapas de la IDE MINAGRI (Figura 2), permite el acceso a las capas de IG que cada una de las instituciones participantes aportan. Actualmente existen más de 600 capas de acceso público con más de 50,000 visitas el último año. También se dispone de más de 900 capas de IG para uso de los profesionales del Ministerio de Agricultura, a las que acceden a través de un acceso especial actualmente registra más de 1,600 usuarios.

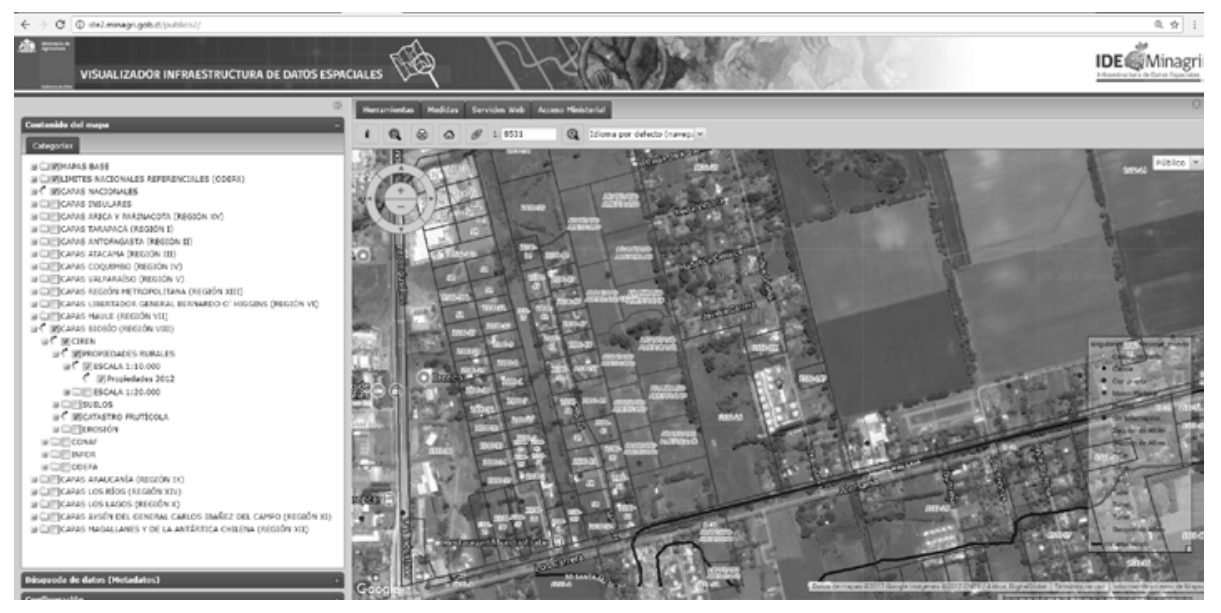

Figura 2. Vista Visualizador de Mapas IDE MINAGRI, $<$ http://ide2.minagri.gob.cl/publico2/>.

Algunas de las capas de IG disponibles en el visualizador de mapas son: propiedades rurales (CIREN), catastro frutícola (CIREN), erosión (CIREN), incendios forestarles (CONAF), catastro de usos del suelo y vegetación (CONAF), zonas 
agroclimáticas, entre otras tantas. La superposición de estas capas permite buscar/localizar, visualizar y analizar IG necesaria para la toma de decisiones, por ejemplo: localizar un determinado predio según su rol; analizar el tipo de suelo en función de sus cualidades agrológicas; determinar las zonas de inversión pública en estructura de riego; conocer las áreas silvestres protegidas por el estado y los recursos forestales del país; obtener información sobre la superficie plantada de las diferentes especies y variedades del país; estadísticas censales silvoagropecuarias y datos de los diferentes pasos fronterizos; etc.

Se han orientado los esfuerzos para que el visualizador de mapas sea sencillo de utilizar para cualquier usuario, simplificando la navegación por medio de la minimización de las herramientas disponibles y se continúa trabajando en la usabilidad del mismo para que responda a los requerimientos de los distintos perfiles de usuario.

El visualizador de mapas dispone de cuatro pestañas: Herramientas, Medidas, Servicios y Acceso Ministerial, es una opción que permite filtrar las herramientas de acuerdo a los distintos requerimientos de los usuarios. A esto se suma la posibilidad de disponer de cuatro tipos de mapas base (Google Maps Híbrido, Google Maps Satelite, Google Maps Terreno y Open Street Map) para que el usuario utilice como base el que responda a sus requerimientos y necesidades de acuerdo a la IG que será objeto de consulta y superposición en el visualizador de mapas. Por otra parte, para evitar los errores de medida en los diferentes tamaños de pantalla se ha añadido la escala gráfica. También desde del visualizador de mapas se puede acceder directamente al Catálogo de Metadatos, lo que permite localizar datos e información relacionada con lo que se está visualizando en la ventana actual del mapa.

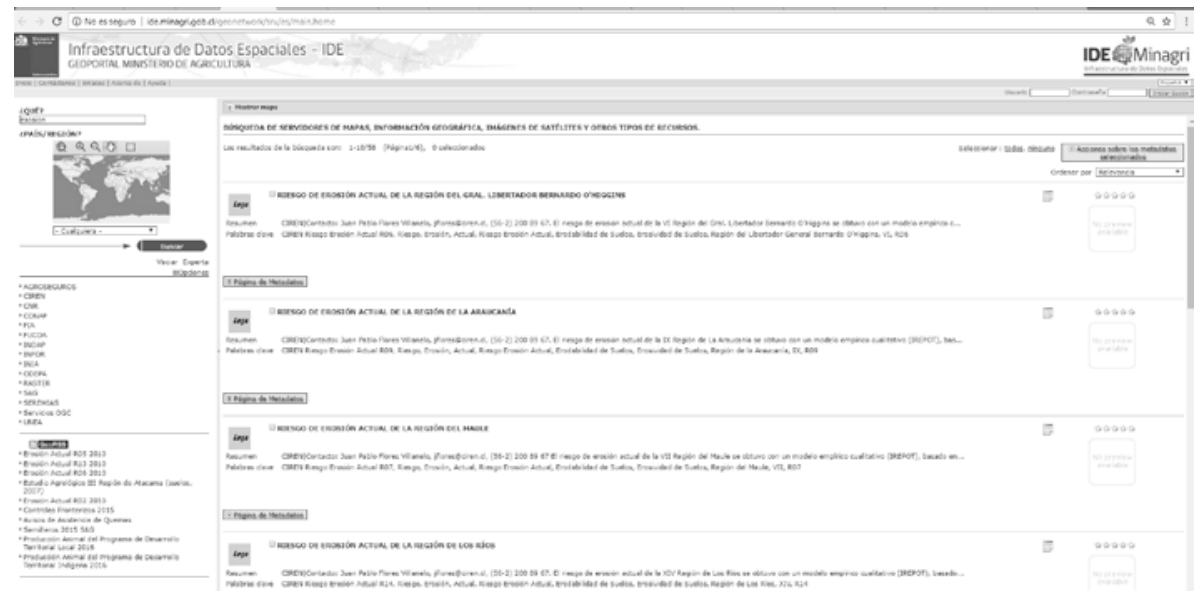

Figura 3. Vista Catálogo de Metadatos IDE MINAGRI, $<$ http://ide.minagri.gob.cl/geonetwork/srv/es/csw $>$. 


\section{Catálogo de metadatos}

Se ha elegido la aplicación de software libre y código abierto Geonetwork para gestionar las búsquedas y acceso a los metadatos. Es una aplicación muy conocida (y reconocida) que de manera fiable permite la búsqueda y el acceso a bases de datos georreferenciadas (Figura 3). En este caso, se accede a los datos que hacen referencia a las características principales de las capas de IG contenidas en la IDE MINAGRI. Al ser un servicio estándar éste puede ser consumido desde otros aplicativos interoperables, por ejemplo, el visualizador de mapas que en su sección de búsqueda de metadatos consume dicho servicio.

La gran ventaja de utilizar Geonetwork es su amplia difusión y por lo tanto su conocido interfaz de uso por lo que se ha preferido no "personalizar" demasiado la herramienta para no desvirtuar su facilidad de uso.

\section{Directorio de servicios}

Esta aplicación permite acceder a los servicios web que están disponibles en la aplicación. Actualmente IDE MINAGRI cuenta con un único servicio WMS que reúne para ser visualizadas todas las capas disponibles que son alrededor de 700. Este servicio es accesible y puede ser consumido desde: $<$ http://ide.minagri.gob.cl/maps/Publico/wms?>.

Los responsables de la IDE MINAGRI han sido conscientes de la dificultad que entraña para los no iniciados en el mundo IDE, la comprensión de lo que se encuentran al entrar en el Directorio de Servicios por lo que un reto a futuro es generar un acceso que permita el uso de los WMS de una manera que responda a la máxima usabilidad. Se es consciente de que no es fácil el uso de ciertas páginas por parte de usuarios no especializados, lo cual dificulta su uso.

\section{Acciones futuras}

Considerando que en último año (2016) se ha producido un notable incremento en los accesos al geoportal IDE MINAGRI (Tabla 1), se continuarán desarrollando distintas estrategias orientadas a potenciar su difusión y uso por distintos perfiles de usuarios.

Tabla 1

Accesos al geoportal IDE MINAGRI, periodo 2014-2016

\begin{tabular}{lc}
\hline Año & Accesos \\
\hline 2014 & 17,650 \\
2015 & 18,590 \\
2016 & 51,908 \\
\hline
\end{tabular}


El desafío es seguir avanzando de forma conjunta con cada uno de los servicios del Ministerio de Agricultura y brindar una información geográfica actualizada y de calidad, contribuyendo así con la democratización de la IG. El objetivo es poder contribuir de manera eficiente en la toma de decisiones tanto por parte de las autoridades ministeriales, como de los profesionales relacionados con ámbito agrícolaganadero y ser una herramienta de apoyo y búsqueda de información para el público en general.

\section{Bibliografía}

Barrera Vielma, M.G. y Guzmán, F. (2016). Proyecto IDE MINAGRI. Taller "Infraestructuras de Datos Espaciales y Usabilidad de Geoportales", Secretaría Ejecutiva del SNIT-IDE Chile, 29 de julio de 2016, Santiago, Chile, $<$ http://www.ide.cl/descargas/20160729-Taller-IDE/IDE-Minagri.pdf>.

Ministerio de Agricultura de Chile (2012). Convenio marco de colaboración para el desarrollo del proyecto denominado "IDE integrada para el Ministerio de Agricultura".

Ministerio de Agricultura de Chile (2016). Resolución Exenta No 124. Aprobación del Convenio Marco de colaboración para el desarrollo denominado "Infraestructura de Datos Espaciales del Ministerio de Agricultura", $<$ http://ide.minagri.gob.cl/geoweb/index.php/recursos/documentos/category/102 -convenios2016>. 